\title{
Segmental Organization of Vestibulospinal Inputs to Spinal Interneurons Mediating Crossed Activation of Thoracolumbar Motoneurons in the Neonatal Mouse
}

\author{
Nedim Kasumacic, ${ }^{1}$ François M. Lambert, ${ }^{1}$ Patrice Coulon, ${ }^{3}$ Helene Bras, ${ }^{3}$ Laurent Vinay, ${ }^{3}$ Marie-Claude Perreault, ${ }^{1,2,4}$ \\ and Joel C. Glover ${ }^{1,2}$ \\ ${ }^{1}$ Laboratory of Neural Development and Optical Recording, Department of Physiology, Institute of Basic Medical Sciences, University of Oslo, N-0317 Oslo, \\ Norway, ${ }^{2}$ Norwegian Center for Stem Cell Research, Oslo University Hospital, N-0372 Oslo, Norway, ${ }^{3}$ Timone Neurosciences Institute, National Center of \\ Scientific Research and Aix-Marseille University, F-13385 Marseille, France, and ${ }^{4}$ Department of Physiology, School of Medicine, Emory University,
} Atlanta, Georgia 30322

Vestibulospinal pathways activate contralateral motoneurons (MNs) in the thoracolumbar spinal cord of the neonatal mouse exclusively via axons descending ipsilaterally from the vestibular nuclei via the lateral vestibulospinal tract (LVST; Kasumacic et al., 2010). Here we investigate how transmission from the LVST to contralateral MNs is mediated by descending commissural interneurons (dCINs) in different spinal segments. We test the polysynaptic nature of this crossed projection by assessing LVST-mediated ventral root (VR) response latencies, manipulating synaptic responses pharmacologically, and tracing the pathway transynaptically from hindlimb extensor muscles using rabies virus (RV). Longer response latencies in contralateral than ipsilateral VRs, near-complete abolition of LVST-mediated calcium responses in contralateral MNs by mephenesin, and the absence of transsynaptic RV labeling of contralateral LVST neurons within a monosynaptic time window all indicate an overwhelmingly polysynaptic pathway from the LVST to contralateral MNs. Optical recording of synaptically mediated calcium responses identifies LVST-responsive ipsilateral dCINs that exhibit segmental differences in proportion and dorsoventral distribution. In contrast to thoracic and lower lumbar segments, in which most dCINs are LVST responsive, upper lumbar segments stand out because they contain a much smaller and more ventrally restricted subpopulation of LVST-responsive dCINs. A large proportion of these upper lumbar LVST-responsive dCINs project to contralateral L5, which contains many of the hindlimb extensor MNs activated by the LVST. A selective channeling of LVST inputs through segmentally and dorsoventrally restricted subsets of dCINs provides a mechanism for targeting vestibulospinal signals differentially to contralateral trunk and hindlimb MNs in the mammalian spinal cord.

Key words: calcium imaging; commissural interneuron; descending motor pathways; spinal network; supraspinal control; vestibular system

\section{Introduction}

The descending control of motoneurons (MNs) in different spinal segments is mediated primarily by segmental and interseg-

Received Dec. 13, 2014; revised March 22, 2015; accepted April 15, 2015.

Author contributions: L.V., M.-C.P., and J.C.G. designed research; N.K., F.M.L., P.C., H.B., and J.C.G. performed research; N.K., F.M.L., P.C., H.B., M.-C.P., and J.C.G. analyzed data; N.K., F.M.L., M.-C.P., and J.C.G. wrote the paper.

This work was supported by grants from the Medical Faculty of the University of Oslo (J.C.G., M.-C.P.), the Norwegian Research Council (J.C.G.), the Southern and Eastern Norway Regional Health Authority (J.C.G.), National Institutes of Health Grant NS085387 (M.-C.P.), French Medical Research Foundation Grant SPE20100518439, and the Partenariat Hubert Curien Aurora (L.V., M.-C.P.). We are grateful to Kobra Sultani and Marian Berge Andersen for technical assistance and Magne Sand Sivertsen for help with figures. We dedicate this article and the work it contains to the memory of our dear colleague and friend Laurent Vinay, who passed away shortly before the manuscript was accepted for publication. His contribution to the field of spinal cord research stands as an enduring example of scientific creativity and integrity.

Correspondence should be addressed to either of the following: Marie-Claude Perreault, Department of Physiology, School of Medicine, Emory University, Whitehead Biomedical Research Building, 615 Michael Street, Atlanta, GA 0322, E-mail: m-c.perreault@emory.edu; or Joel C. Glover, Laboratory of Neural Development and Optical Recording, Department of Physiology, Institute of Basic Medical Sciences, University of Oslo, N-0317 Oslo, Norway. E-mail:joel.glover@medisin.uio.no.

DOI:10.1523/JNEUROSCI.5188-14.2015

Copyright $\odot 2015$ the authors $\quad 0270-6474 / 15 / 358158-12 \$ 15.00 / 0$ mental interneurons (INs), but how these channel and distribute descending inputs to different $\mathrm{MN}$ targets is only superficially understood. The vestibulospinal system, which plays a central role in the control of posture and movement, provides an example. The lateral vestibulospinal tract (LVST) has long been known to coordinate trunk and limb movements (Lund and Pompeiano, 1968; Wilson and Yoshida, 1969; Grillner et al., 1970; Wilson et al., 1970; Hongo et al., 1971; Grillner and Hongo, 1972; Abzug et al., 1974; Kasumacic et al., 2010), but information about the connectivity of LVST axons to different spinal IN populations is only fragmentary. We demonstrated recently LVST-mediated inputs to axial and limb MNs in the medial (MMC) and lateral (LMC) motor columns, respectively, in cervical, thoracic, and lumbar segments in the neonatal mouse (Kasumacic et al., 2010). In lumbar segments, LVST-mediated inputs were targeted contralaterally to both MMC and LMC MNs but ipsilaterally only to LMC MNs. This concerted yet asymmetric action of LVST-mediated inputs on lumbar axial and limb MNs provides a potential substrate for the trunk-limb coordination required to maintain balance and regulate posture during motor tasks. 
LVST axons descend only ipsilaterally, and their activation of contralateral lumbar MNs depends on an intact lumbar commissure (Kasumacic et al., 2010). Thus, it must be mediated by some combination of locally crossing LVST axon collaterals, midlinecrossing MN dendrites, and excitatory commissural INs (CINs). Some LVST axon collaterals have been shown to cross the midline in adult cats and mice, but this is uncommon in the lumbar cord (Kuze et al., 1999; Liang et al., 2014). Some MMC MN populations have midline-crossing dendrites (Rose and Collins, 1985; Huang and Goshgarian, 2009). Stimulation of the lateral vestibular nucleus, from which most LVST axons originate, elicits monosynaptic and disynaptic responses in last-order premotor ipsilateral CINs in L4-L5 of the adult cat (Krutki et al., 2003). Although these findings indicate a prominent role for CINs in mediating the crossed LVST pathway, little information is available about the intrasegmental and intersegmental distribution of LVST-responsive CINs and how this relates to activation of contralateral trunk and hindlimb MNs.

Our goal here was to assess in the newborn mouse whether the pathway from LVST to contralateral MNs is polysynaptic, test for functional vestibulospinal connections to descending CINs (dCINs; Eide et al., 1999; Nissen et al., 2005), define the distribution of LVST-responsive dCINs in different thoracolumbar segments, and assess the segmental extent of axonal projections of responsive dCINs toward potential lumbar MN targets. We document a polysynaptic pathway to contralateral $\mathrm{MNs}$ and a segment-specific partitioning of LVST-responsive from LVSTnonresponsive ipsilateral dCINs. The segmentally patterned organization provides a means for a selective channeling of LVST inputs to different contralateral trunk and hindlimb MNs and also suggests a potential developmental mechanism for securing it. This hitherto unappreciated segmental patterning of descending inputs to spinal INs may represent a general organizational feature of mammalian descending motor systems.

\section{Materials and Methods}

Animals. Rabies virus (RV) experiments were performed on newborn mice of the OF1 strain $(n=14)$, and all other experiments were performed on ex vivo preparations of the brainstem and spinal cord of newborn mice of the ICR strain $(n=65)$ at postnatal day $0(\mathrm{P} 0)$ to P5. Because gender determination is difficult in newborns, experiments were necessarily performed on either gender. All efforts were made to minimize the number of animals used in accordance with the European Communities Council directive 86/609/EEC and the National Institutes of Health Guidelines for the Care and Use of Animals. All procedures were approved by the National Animal Research Authority in Norway (Forsøksdyrutvalget, Permit 2762) and by the Ministry for Agriculture and Fisheries, Division of Animal Rights in France and the local safety committee at the University of Marseille (Permit 02167-02).

Transsynaptic retrograde tracing with $R V$. Newborn mice were anesthetized by hypothermia. A small skin incision was made at the level of the right triceps surae (TS) muscle group. An injector cannula (33 gauge; Plastics One) connected to a $10 \mu$ l Hamilton syringe with a catheter $(0.2$ $\mathrm{mm}$ outer diameter) was inserted into the muscle. RV (challenge virus standard-N2C strain; $0.6 \mu \mathrm{l} ; 1.6 \times 10^{6} \mathrm{pfu}$ ) was injected slowly according to the procedure described by Bras et al. (2008). In P1 animals, the small size prevented us from restricting the injection site or the spread of the inoculum to individual muscles of the TS muscle group. However, all injections were performed by the same person to minimize variability. Because the distribution of RV-infected MNs along the L4-L5 segments was reproducible and fit the pattern published previously in a study of last-order premotor INs (Coulon et al., 2011), it likely reflects a reliable restriction of the inoculum to the TS muscle group.

Ex vivo brainstem-spinal cord preparation. Mice were anesthetized deeply with isoflurane (Abbott Labs) and decerebrated at the level of the superior colliculus, and the brainstem and spinal cord were dissected rapidly and removed using the procedure described by Kasumacic et al. (2010). Preparations were maintained in oxygenated artificial CSF (ACSF; in mM: $128 \mathrm{NaCl}, 3 \mathrm{KCl}, 11 \mathrm{D}$-glucose, $2.5 \mathrm{CaCl}_{2}, 1 \mathrm{MgSO}_{4}, 1.2$ $\mathrm{NaH}_{2} \mathrm{PO}_{4}, 5$ HEPES, and $25 \mathrm{NaHCO}_{3}$ ).

Retrograde tracing with rhodamine- and biotin-conjugated dextranamines. Retrograde labeling of the LVST (in preparations from animals in which RV was used for transsynaptic retrograde tracing from the TS muscle group) or dCINs was performed with rhodamine-conjugated dextran-amine [RDA; 3000 molecular weight (MW); Invitrogen] and biotin-conjugated dextran-amine (BDA; $3000 \mathrm{MW}$; Invitrogen), as described previously (Glover et al., 1986; Glover, 1995; Stokke et al., 2002; Nissen et al., 2005; Kasumacic et al., 2010). To label the LVST, a unilateral transverse cut was made through the ventral funiculus (VF) and ventrolateral funiculus (VLF) at the C1 level, and several crystals of a mixture of RDA and BDA were applied sequentially to the cut to maintain a high concentration for $3 \mathrm{~min}$. The preparations were incubated in oxygenated ACSF at room temperature (RT) in the dark for 8-10 h and then fixed with $4 \%$ paraformaldehyde (PFA) in PBS, $\mathrm{pH}$ 7.4. For differential labeling of dCINs in L2 with different longitudinal axon lengths, a unilateral transverse cut was first made in the VF and VLF at the L4/L5 border, to which BDA crystals were applied as described above. After incubation in oxygenated ACSF for 3-4 h at RT in the dark, a second transverse cut was made in the VF and VLF at the L2/L3 border, to which RDA crystals were applied as described above. After additional incubation in oxygenated ACSF for 3-4 h at RT in the dark, the preparations were fixed with $4 \%$ PFA.

Immunohistochemistry and streptavidin-biotin reaction. Brainstems or spinal cords were cryoprotected with $20 \%$ and then $30 \%$ sucrose in PBS and freeze embedded in OCT (optimum cutting temperature) compound (VWR Scientific). Immunohistochemistry and streptavidin-biotin reaction were performed on 14 or $30 \mu \mathrm{m}$ cryostat sections collected onto poly-L-lysine-coated slides. Briefly, sections were blocked for unspecific binding for at least $30 \mathrm{~min}$ in blocking solution [10\% normal goat serum, $1 \%$ nonfat milk powder, and $0.02 \%$ Na-azide in Trisbuffered saline containing $0.5 \%(\mathrm{w} / \mathrm{v})$ Tween 20 (TBST), pH 7.4]. Sections from RV-labeled preparations were then incubated overnight at $4^{\circ} \mathrm{C}$ with primary antibody to RV phosphoprotein (specificity described previously by Coulon et al., 2011) diluted 1:10,000 in blocking solution. The sections were rinsed five times for 5 min with PBS, followed by a single rinse with TBST and then incubated overnight at $4^{\circ} \mathrm{C}$ with 1:200 Cy3-conjugated streptavidin (catalog \#016-160-084; Jackson ImmunoResearch) and 1:400 Alexa Fluor 488-conjugated goat anti-mouse IgG (catalog \#A-11017; Invitrogen). The sections were rinsed five times for 5 min with PBS, counterstained with the nuclear stain Hoechst 33258 (1 $\mu \mathrm{g} / \mathrm{ml}$ in PBS) for $1 \mathrm{~min}$, mounted under coverslips with PBS-glycerol at $1: 1$, and examined under fluorescence optics.

Fluorescence microscopy. Sections from transsynaptic retrograde RV and retrograde dextran-amine labeling were imaged at $10 \times$ and $20 \times$ with a Leica DMRB epifluorescence microscope using FITC and $568 \mathrm{~nm}$ filters. Images were processed offline using NIH Image J freeware.

Electrophysiology. Unilateral activation of the LVST was achieved by electrical stimulation of the VIIIth cranial nerve. We have shown previously in the neonatal mouse that, of the vestibulospinal projections, only the LVST, not the medial vestibulospinal tract (MVST), projects to thoracic and lumbar levels (Kasumacic et al., 2010). We have also shown, through lesion experiments and comparison with stimulation of the reticular formation, that responses in lumbar MNs to stimulation of the VIIIth nerve are mediated exclusively ipsilaterally, with no contribution from crossed connections in the brainstem, and predominantly by the ipsilateral LVST, with little if any contribution from ipsilateral reticulospinal projections (Kasumacic et al., 2010). Thus, we consider responses in the lower thoracic and lumbar region of the cord to stimulation of the VIIIth nerve essentially to be mediated selectively by the ipsilateral LVST, although we cannot rule out a very minor reticulospinal contribution. Electrical stimulation of the VIIIth cranial nerve or lumbar dorsal roots (DRs) was performed as described by Kasumacic et al. (2010) and Boulland et al. (2013). Briefly, fire-polished borosilicate glass (1.2 mm outer diameter, $0.94 \mathrm{~mm}$ inner diameter; catalog \#30- 
0056; Harvard Apparatus) suction electrodes with appropriately sized tip diameters were used to deliver short $(0.2-0.5 \mathrm{~ms})$ electrical current pulses using one of two different stimulators (DS8000 from WPI or S48 from Grass Instruments). Either single pulses (at least $50 \mathrm{~s}$ between two consecutive pulses) or pulse trains ( $5 \mathrm{~Hz}$ for $5 \mathrm{~s}$, at least $3 \mathrm{~min}$ between trains) were used for both electrophysiological and optical recording experiments. Current strengths ranged from 40 to $500 \mu \mathrm{A}$ (VIIIth cranial nerve) and 10 to $20 \mu \mathrm{A}$ (DRs), and, in experiments in which calcium responses were recorded, the current strengths were expressed as multiples of the current threshold ( $\mathrm{T}$ ) for eliciting a detectable calcium response (see below).

Extracellular recording of activity in lumbar ventral roots (VRs) was performed as described by Brocard et al. (1999) and Boulland et al. (2013). Briefly, fire-polished borosilicate glass suction recording electrodes with appropriately sized tip diameters were placed en passant onto lumbar VRs a short distance from the root exit site to record compound action potentials representing $\mathrm{MN}$ discharges. Electrical signals were amplified (EXT 10-2F amplifier; npi Electronics), digitized (10 kHz), averaged, and analyzed offline (CED 1401 mkII, SPIKE 2; Cambridge Electronic Design). Electrophysiological recordings of compound action potentials were rectified and integrated with SPIKE 2 software (time constant, $1 \mathrm{~ms}$ ). Onset latency was calculated by measuring the time between the onset of the first stimulus pulse and the onset of the first upward slope that exceeded 2 SDs above the mean baseline noise in the rectified/integrated trace of the VR recording. In all preparations, onset latencies were measured from responses of 7-10 consecutive single pulses and averaged to provide mean latencies.

Optical recording of synaptically mediated calcium responses. MNs were retrogradely labeled by applying crystals of Calcium Green 1-conjugated dextran-amine (CGDA; $3000 \mathrm{MW}$; Invitrogen) to cut VRs, and dCINs were retrogradely labeled by applying CGDA to a transverse cut through the VF and VLF one segment caudal to the segment of recording. Retrograde labeling was allowed to continue in ACSF at RT in the dark for $4 \mathrm{~h}$ before recording. Preparations were then transferred to a Sylgard-coated chamber and pinned down with stainless steel (Minuten) pins ventral side up. The bath containing the brainstem-spinal cord preparation was perfused with oxygenated ACSF at a rate of $7.5 \mathrm{ml} / \mathrm{min}$, giving a total volume exchange every $2 \mathrm{~min}$. Labeled MNs were visualized from the ventral surface, and labeled dCINs were visualized from the face of an oblique cut made through the recorded segment (Szokol and Perreault, 2009; Kasumacic et al., 2012) using a $40 \times$ water-immersion objective (LUMPlanFl, 0.8 numerical aperture; Olympus) on an upright epifluorescence microscope (Axioskop FS 2; Carl Zeiss) equipped with a $100 \mathrm{~W}$ halogen lamp driven by a direct current power supply (PAN35-20A; Kikusui Electronics) and excitation and emission filters $(450-490 \mathrm{~nm}$ bandpass and $515 \mathrm{~nm}$ long pass, respectively). Fluorescence changes associated with synaptically mediated events, which we refer to hereafter as "calcium responses," were registered using a cooled CCD camera (Cascade 650 or Evolve:512; Photometrics, Texas Instruments) mounted on a video zoom adaptor, using the image-processing software MetaMorph 5.0 (Universal Imaging, Molecular Devices). Image series were acquired at 4 frames/s (fps, binning factor of 2) or $150 \mathrm{fps}$ (binning factor of 6). In experiments in which we compared the magnitude of a response in a given cell before, during, and after pharmacological intervention, imaging series were acquired at 4 fps.

Plotting dCIN locations in transverse sections. After calcium recording, a series of images was taken of adjacent regions of the surface of the oblique cut spinal cord, and the individual images were tiled to construct a complete image of the entire cut surface. This image was used to make a schematic diagram of the oblique face as shown in Figure 6. Responsive and nonresponsive neurons were identified from the recordings and marked on the full-view image. These marks were then transferred to the schematic section.

Pharmacological manipulation with mephenesin. The spinal cord was first isolated from the brainstem with a Vaseline barrier at the C1 level and then superfused with mephenesin (1 mM in ACSF; Sigma) at the same rate as for ACSF superfusion. The tightness of the barrier was verified at the end of the experiment by adding the dye fast green to either the spinal or brainstem compartment. Recordings started $10 \mathrm{~min}$ after drug application. Washing out started $20 \mathrm{~min}$ after mephenesin application and lasted at least $10 \mathrm{~min}$.

Analysis of optical records. Circular digital apertures of identical size and shape, termed regions of interest (ROIs) in the MetaMorph software, were manually placed over individual $\mathrm{MN}$ or $\mathrm{dCIN}$ somata. To compensate for variability in the CGDA labeling intensity, the calcium responses in each ROI were quantified as changes in fluorescence, $\Delta F$, divided by the baseline fluorescence, $F_{0}$, before the stimulation $\left[\Delta F / F_{0}=\left(F-F_{0}\right) / F_{0}\right]$. A detectable response was defined as a continuous positive deflection with an onset defined as the time point at which the deflection exceeded a detection limit of 2 SDs above the mean of the baseline. Response latency was defined as the difference in time between stimulation onset and the onset of a detectable response. Response magnitude was defined as the area under the waveform above the detection limit. Unless indicated otherwise, all data are presented as grand means across preparations.

Pseudocolor representations of responses were made by filtering the complete image series of the recording session in MetaMorph with a low-pass $3 \times$ 3 filter and then converting grayscale values to colors using a rainbow index, with transition from blue to red to white representing increasing response size. In some cases, adjustments in brightness and contrast were performed on the entire image series before this conversion using the auto-levels function to avoid loss of data attributable to saturation.

Statistics. Data are presented as means and SEMs, unless stated otherwise. For both electrophysiological and optical recording data, differences of means were tested by using the nonparametric unpaired Mann-Whitney $U$ test (significance threshold at 5\%). Statistics were performed with OriginPro 8 and SigmaPlot 11.0 software.

\section{Results}

LVST-mediated inputs to contralateral MNs are polysynaptic We have shown previously that calcium responses in lumbar MNs to stimulation of the VIIIth nerve are mediated predominantly if not exclusively by the ipsilateral LVST, with no contribution of crossed connections in the brainstem (Kasumacic et al., 2010; see Materials and Methods). Therefore, we use the term "LVST-mediated" to denote all calcium responses to VIIIth nerve stimulation in neurons of the lower thoracic and lumbar cord here. We have also shown previously that a midline lesion encompassing L1-L6 eliminates LVST-mediated calcium responses in contralateral MNs in segments L2 and L5 (Kasumacic et al., 2010), suggesting that locally crossing excitatory connections play an important role in mediating these responses. To test the degree of polysynapticity in this pathway, we performed three different types of experiments.

First, we measured the latency of LVST-mediated responses in both VRs of L5 $(n=8$; Fig. $1 A)$. We selected L5 specifically because it contains mainly knee and ankle extensor MNs (McHanwell and Biscoe, 1981; but see Cowley and Schmidt, 1994; Bácskai et al., 2014), the principal targets of the monosynaptic inputs from the LVST in the adult cat (Grillner and Hongo, 1972). Indicative of different numbers of interposed synapses, LVST-mediated responses in the contralateral L5 VR had clearly longer latencies than those in the ipsilateral L5 VR $(91.7 \pm 2.5 \mathrm{vs}$ $63.8 \pm 1.0 \mathrm{~ms}$, respectively; Mann-Whitney $U$ test, $p<0.001$; Fig. $1 B$ ). To place this in perspective to another source of input to MNs, characterized previously in the neonatal rat (Juvin and Morin 2005), we compared the LVST-mediated response latencies with those obtained after stimulation of the DR. As shown in Figure 1C, L5 DR-evoked responses in the contralateral L5 VR, which are at least disynaptic, had a latency of $15.0 \pm 0.2 \mathrm{~ms}, \sim 9$ $\mathrm{ms}$ longer than the monosynaptic responses in the ipsilateral L5 VR $(6.3 \pm 0.1 \mathrm{~ms})$. This indicates that the difference between LVST-mediated response latencies in MNs on the two sides ( $\sim 30$ ms on average) is sufficient to accommodate at least one extra 
A

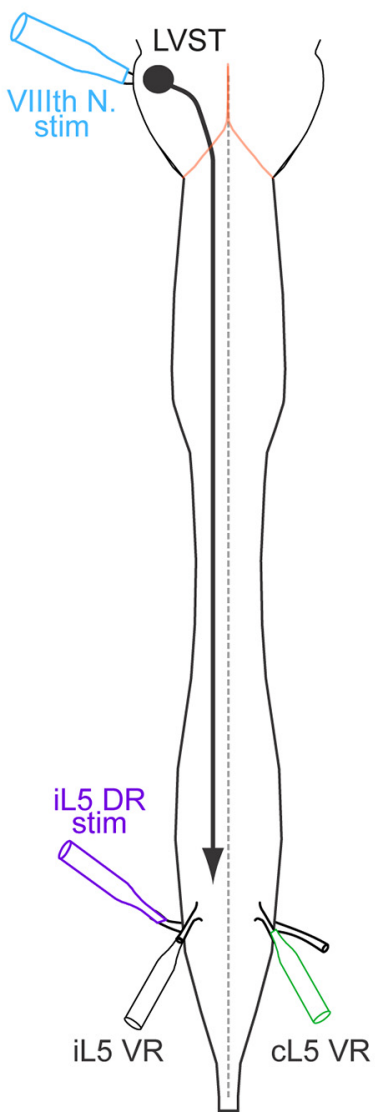

B

VIIIth N.

stim

iL5 VR

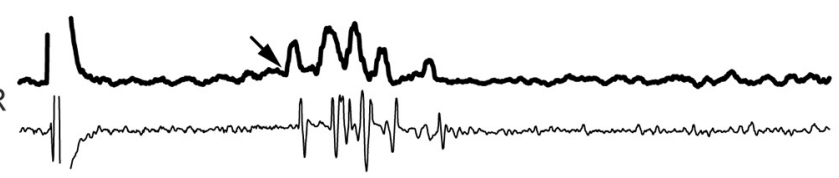

CL5 VR

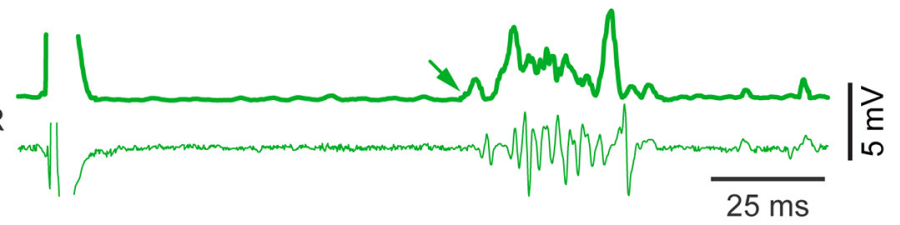

C

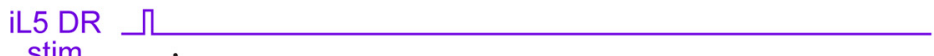

stim
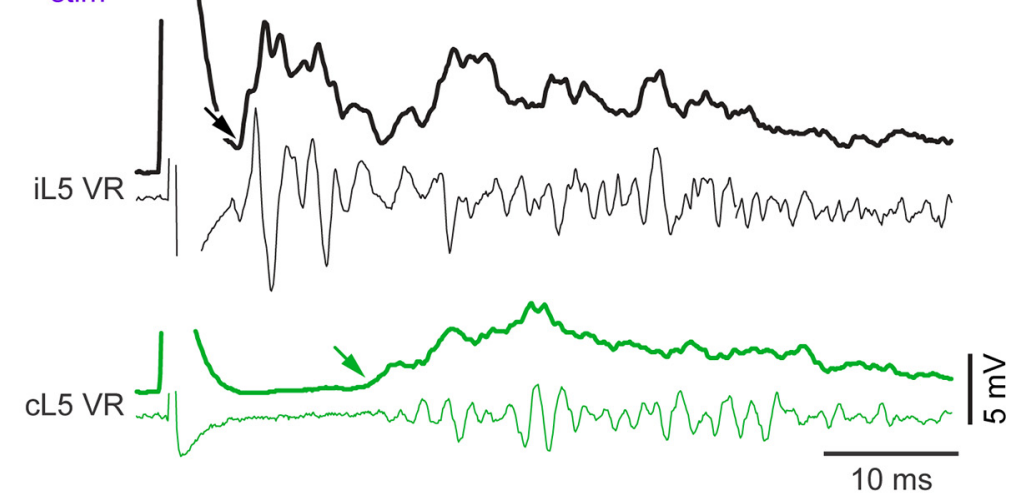

Figure 1. LVST-mediated compound action potentials in ipsilateral and contralateral L5 VRs. A, Schematic overview of the brainstem-spinal cord preparation used to record electrophysiological compound action potentials in ipsilateral and contralateral lumbar VRs (iL5 and cL5 VR) in response to electrical stimulation of the VIIIth cranial nerve (VIIIth N. stim) or of the segmental DR (iL5 DR stim). The bottom trace in each recording shows raw recording, and the top thicker trace shows rectified/integrated signal (arrows indicate the response onset). $\boldsymbol{B}$, Compound action potentials recorded in iL5 VR and CL5 VR in response to a single $500 \mu$ s stimulation pulse to the VIIIth nerve. The difference in response onset in this example is $38.5 \mathrm{~ms}$. C, Compound action potentials recorded in iL5 VR and CL5 VR in response to a single $500 \mu$ s stimulation pulse to the iL5 DR. The difference in response onset in this example is $10.8 \mathrm{~ms}$.

synapse in the pathway from LVST to contralateral MNs relative to ipsilateral MNs.

As a second test of polysynapticity, we examined the effects of $1 \mathrm{~mm}$ mephenesin on LVST-mediated calcium responses in lumbar MNs. Mephenesin, known to preferentially reduce polysynaptic transmission in sensory (Ziskind-Conhaim, 1990; Lev-Tov and Pinco, 1992; Juvin and Morin, 2005) and descending (Floeter and Lev-Tov, 1993; Vinay et al., 1995; Kasumacic et al., 2010, 2012) pathways, was applied selectively to the spinal cord (Fig. 2A). As shown in Figure 2B1, within 10 min of mephenesin application, LVST-mediated responses in contralateral L5 LMC MNs virtually disappeared (bottom traces), whereas a residual, presumably monosynaptic, response in ipsilateral MNs remained (top traces). The effect was reversible. As a positive control, we assessed monosynaptic and polysynaptic responses in ipsilateral L5 MNs to stimulation of the L5 DR and observed an effect of mephenesin entirely comparable to that seen in previous publications (Fig. $2 \mathrm{~B}_{2}$ ). Mephenesin had a similar effect on LVSTmediated responses in all contralateral MNs in L2 and L5 ( $n=3$ animals), including those in the MMC (Fig. 2C). Response magnitudes in the different contralateral $\mathrm{MN}$ populations decreased by $>90 \%$ (percentage of control response remaining: $5.8 \pm 3.9$ in contralateral L2 MMC; $6.7 \pm 4.9$ in contralateral L2 LMC; $2.1 \pm$ 1.0 in contralateral L5 MMC; $1.0 \pm 0.7$ in contralateral L5 LMC), whereas a larger residual response remained in the ipsilateral L2 and L5 LMC (percentage of control response: $16.3 \pm 3.3$ and
$16.0 \pm 2.1$, respectively; there are no LVST-mediated responses in ipsilateral MMC MNs; Fig. 2D).

As a third test of polysynapticity, specifically designed to determine whether any LVST neurons have direct synaptic connections to contralateral lumbar MNs, we used retrograde transsynaptic tracing with RV. For these experiments, we injected RV into the right TS ankle extensor muscle group at P1. After different incubation times, we then retrogradely labeled the entire population of ipsilaterally and contralaterally projecting vestibulospinal axons with RDA/BDA applied at $\mathrm{C} 1$ on the right side (Fig. 3, top left diagram).

In preliminary experiments testing RV incubation times ranging from 30 to $48 \mathrm{~h}$ ( $n=8$ mice), we determined that right LVST neurons become RV labeled sometime between 30 and $38 \mathrm{~h}$ after infection. At $30 \mathrm{~h}$, no LVST neurons, or hindbrain neurons of any type, were RV labeled, but many spinal INs were clearly RV labeled in a pattern resembling that which we have reported previously after injection of RV into the TS muscle group (Coulon et al., 2011). By 38-42 h, substantial numbers of right LVST neurons were RV labeled, and a few left LVST neurons were very faintly RV labeled. At $48 \mathrm{~h}$, greater numbers of both right and left LVST neurons were RV labeled, as were neurons in the deep cerebellar nuclei and Purkinje neurons on the right side, both of which are presynaptic to LVST neurons ipsilaterally.

Based on these preliminary experiments, we injected RV into a second group of mice $(n=6)$ in which retrograde labeling with 
A

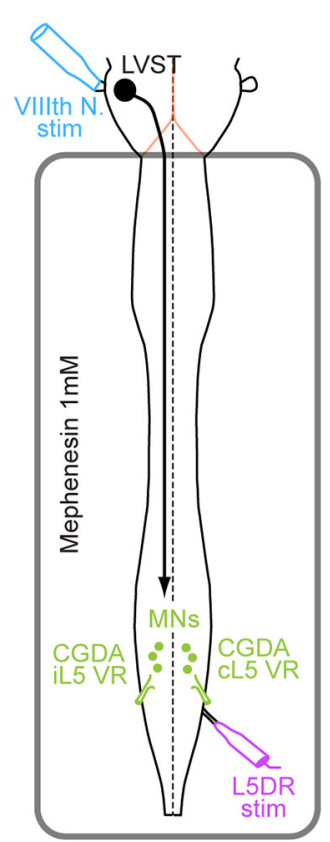

C

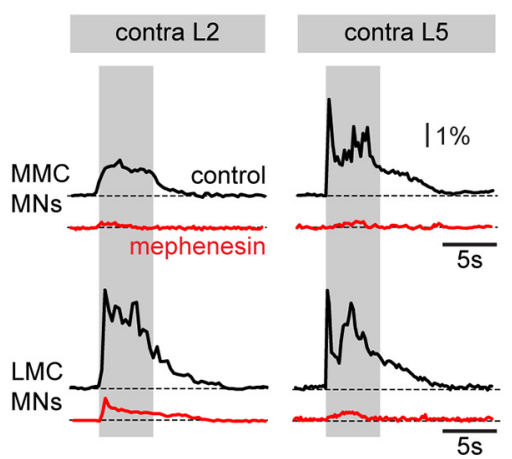

B1
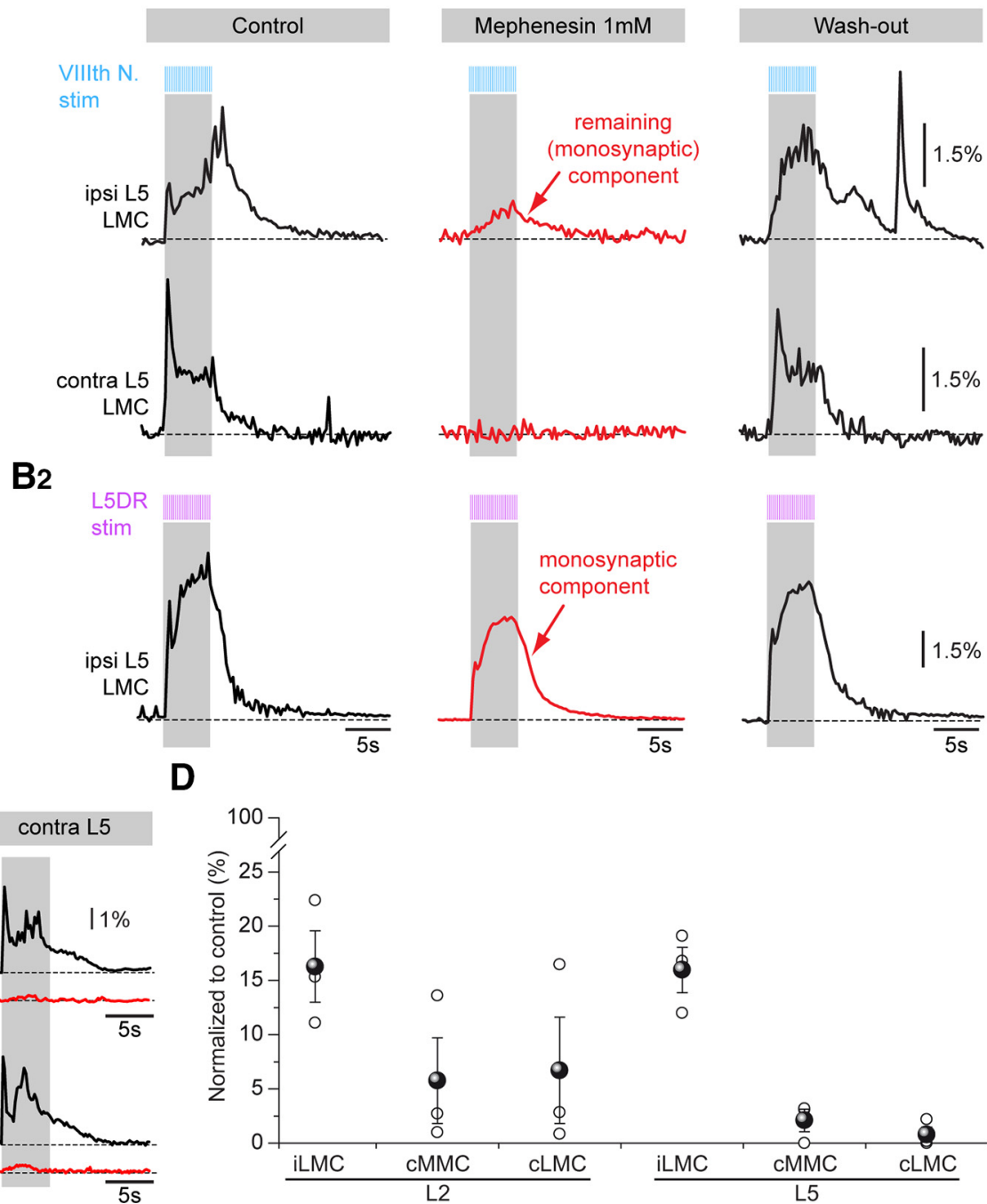

Figure 2. Effect of mephenesin on LVST-mediated calcium responses in lumbar MNs. A, Schematic overview of the brainstem-spinal cord preparation used to optically record calcium transients in ipsilateral and contralateral lumbar MNs in response to electrical stimulation of the VIIIth nerve (VIIIth N. stim). The smooth-cornered rectangle represents the split bath used to isolate and superfuse the spinal cord selectively with $1 \mathrm{~mm}$ mephenesin. $\boldsymbol{B}$, Calcium responses (recorded at $4 \mathrm{fps}$ ) in ipsilateral (ipsi) and contralateral (contra) L5 MNs (iL5 and CL5) from the LMC in response to VIIIth nerve stimulation $\left(\boldsymbol{B}_{1}\right)$ or to L5 DR stimulation (L5 DR stim; $\boldsymbol{B}_{2}$ ) during control (left, black traces), mephenesin application (middle, red traces), and after washout (right, black traces) conditions. C, Calcium responses (recorded at $4 \mathrm{fps}$ ) in contralateral L2 LMC and MMC and L5 MMC MNs in response to VIIIth nerve stimulation during control (top, black traces) and mephenesin application (bottom, red traces) conditions. Gray bars in $\boldsymbol{B}$ and $\boldsymbol{C}$ represent the $5 \mathrm{~s}, 5 \mathrm{~Hz}$ stimulation train. $\boldsymbol{D}$, Percentages of residual LVST-mediated responses (normalized to control responses) in lumbar MNs during mephenesin application. Black spheres and bars represent mean $\pm \mathrm{SEM}$, respectively. White circles represent values from individual preparations.

RDA/BDA was performed at $34 \mathrm{~h}$ after infection. In these mice, no contralateral LVST neurons were RV labeled (Fig. 3A), but a small proportion of ipsilateral LVST neurons ( $7 \pm 3 \%$ ) was consistently RV labeled (Fig. 3B,C). In our previous study, we showed that RV transsynaptically labels neurons in the spinal cord in a retrograde sequence at about $12 \mathrm{~h}$ intervals, with TS MNs labeled first at $18 \mathrm{~h}$ and last-order premotor spinal INs labeled first at $30 \mathrm{~h}$ after injection (Coulon et al., 2011). Therefore, we interpret the initial RV labeling of the small proportion of right LVST neurons at $34 \mathrm{~h}$ to indicate that these are LVST neurons with monosynaptic connections onto right TS MNs. Because substantial numbers of both right and left LVST neurons were RV labeled by $48 \mathrm{~h}$, we interpret these to be disynaptically connected to the right TS MNs. Thus, most connections from LVST neurons to ipsilateral TS MNs and virtually all connections from LVST neurons to contralateral TS MNs are disynaptic or higher-order polysynaptic. The few left LVST neurons that were faintly RV labeled within the time window between $34 \mathrm{~h}$ (when the first ipsilateral right LVST neurons became RV labeled) and $48 \mathrm{~h}$ (when substantial numbers of contralateral left LVST neurons were strongly RV labeled) we interpret to represent a small minority of LVST neurons that make monosynaptic connections onto contralateral TS MNs through midline-crossing axon collaterals (see Discussion).

Together with our previous lesion experiments (Kasumacic et al., 2010), these electrophysiological, pharmacological, and $\mathrm{RV}$-tracing experiments indicate that LVST-mediated inputs to contralateral lumbar MMC and LMC MNs are primarily polysynaptic and thus channeled through spinal CINs.

\section{Optical recording of LVST-mediated synaptic input to ipsilateral dCINs}

To test specifically the potential role of CINs in mediating LVSTmediated excitation of contralateral MNs, we tested for the presence of LVST-mediated synaptic inputs in 482 ipsilateral dCINs in thoracic and lumbar segments of 22 animals (Fig. 4, Table 1; 83 dCINs in T7, 87 dCINs in L1, 163 dCINs in L2, and 149 dCINs in 


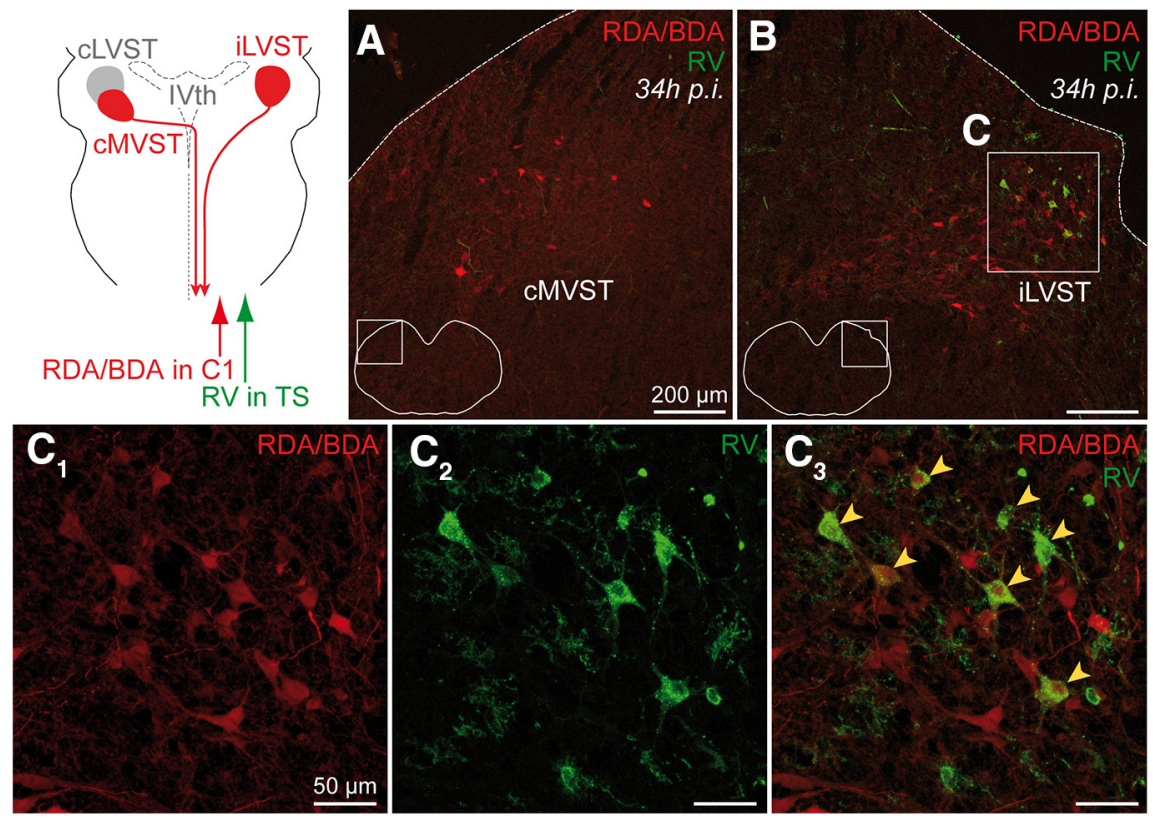

Figure 3. LVST neurons presynaptic to hindlimb extensor MNs identified by transsynaptic retrograde tracing with RV. The top left shows a schematic overview of the location of the two main vestibulospinal groups, the ipsilateral LVST (iLVST) and contralateral MVST (cMVST) groups (both red), which were retrogradely labeled by RDA/BDA application at C1 on the right side (red arrow). Also shown is the unlabeled LVST group on the opposite side (CLVST, gray domain) and the side (right) on which RV was injected into the TS muscle group of the hindlimb (green arrow). IVth V, Fourth ventricle. $\boldsymbol{A}$, Fluorescence micrograph of a transverse section through the brainstem $34 \mathrm{~h}$ after RV injection, showing cMVST neurons in the left vestibular region labeled with RDA/BDA (red). No RV-labeled neurons were detected in the left vestibular region at $34 \mathrm{~h}$. The inset shows the location of image within the section. $\boldsymbol{B}$, Fluorescence micrograph of a transverse section showing RDA/BDA-labeled (red) and RV-labeled (green) iLVST neurons in the right vestibular region. The inset shows the location of image within the section. $\boldsymbol{C}$, Higher-magnification image of the boxed area in $\boldsymbol{B}$, showing RDA/BDA-labeled LVST neurons $\left(\boldsymbol{C}_{\boldsymbol{1}}\right.$, red), RV-labeled LVST neurons ( $\boldsymbol{C}_{2}$, green), and RDA/BDA/RV-double-labeled LVST neurons ( $\boldsymbol{C}_{\boldsymbol{3}}$, yellow arrowheads). p.i., Postinjection. Scale bars: $200 \mu \mathrm{m}(\boldsymbol{A}, \boldsymbol{B}), 50 \mu \mathrm{m}\left(\boldsymbol{C}_{\mathbf{1}}, \boldsymbol{C}_{\mathbf{2}}, \boldsymbol{C}_{\mathbf{3}}\right)$.

L5). In T7 and L5, $>90 \%$ of the ipsilateral dCINs examined responded to activation of the LVST pathway. In contrast, in L1 and L2, only $\sim 40$ and $35 \%$ of the dCINs were responsive, respectively (Fig. 4C, Table 1).

Because T7, L1, L2, and L5 dCINs were recorded in different preparations, we tested whether the lack of LVST-mediated responses in the majority of L1 and L2 dCINs was genuine by determining whether the nonresponsive dCINs were able to respond to other sources of inputs. All LVST nonresponsive dCINs responded to stimulation of the ipsilateral VF at C6 (which activates multiple descending pathways) or of the ipsilateral L2 DR (which activates multiple sensory pathways; Fig. 4D).

To assess the relative timing of activation of ipsilateral dCINs compared with contralateral MNs, we recorded LVST-mediated calcium responses in a sample of the responsive ipsilateral dCINs in T7 ( $n=4$ animals, $37 \mathrm{dCINs),} \mathrm{L2} \mathrm{(} n=6$ animals, $53 \mathrm{dCINs}$ ), and L5 ( $n=6$ animals, $47 \mathrm{dCINs)}$ and in contralateral MNs in T7 ( $n=4$ animals, $35 \mathrm{MNs}$, all in MMC), L2 ( $n=4$ animals, $27 \mathrm{MNs}$ in LMC and $25 \mathrm{MNs}$ in MMC), and L5 MNs ( $n=3$ animals, 24 $\mathrm{MNs}$ in LMC, $18 \mathrm{MNs}$ in MMC) using high-frame rate recording (150 fps; Fig. 5A, $B$ ). Response latencies of the ipsilateral dCINs ranged from 32 to $140 \mathrm{~ms}$ [mean, $76 \pm 3 \mathrm{~ms}$; interquartile range (IQR), 67-82 ms] in T7, 32-116 ms (mean, $73 \pm 3 \mathrm{~ms}$; IQR, $58-88 \mathrm{~ms}$ ) in L2, and 56-130 ms (mean, $86 \pm 3 \mathrm{~ms}$; IQR, 72-98 $\mathrm{ms}$ ) in L5. In each segment, a majority of dCIN responses occurred within $80 \mathrm{~ms}$ (70, 63, and 51\% in T7, L2, and L5 dCINs, respectively). These response latencies were significantly shorter than the response latencies in contralateral MNs (Fig. $5 C-F$ ). In T7, response latencies in contralateral MNs (all in MMC) ranged from
57 to 121 ( $85 \pm 3$ ms; IQR, 77-98 ms). In L2, response latencies in contralateral $\mathrm{MNs}$ ranged from 77 to $313 \mathrm{~ms}$ in MMC (mean, $156 \pm 18 \mathrm{~ms}$; IQR, 88-235 ms) and from 67 to $381 \mathrm{~ms}$ in LMC (mean, $183 \pm 19 \mathrm{~ms}$; IQR, 88-266 ms). In L5, response latencies in contralateral MNs ranged from 57 to 224 $\mathrm{ms}$ in MMC (mean, $127 \pm 13 \mathrm{~ms}$; IQR, 83$182 \mathrm{~ms}$ ) and from 46 to $153 \mathrm{~ms}$ in LMC (mean, $99 \pm 5 \mathrm{~ms}$; IQR, 88-110 ms). In each segment, a majority of contralateral $\mathrm{MN}$ responses occurred after $80 \mathrm{~ms}(60,79$, and $75 \%$ in $\mathrm{T} 7, \mathrm{~L} 2$, and $\mathrm{L} 5 \mathrm{MNs}$, respectively, MMC and LMC pooled).

These findings show that dCINs are activated by the LVST with response latencies compatible with a role in mediating polysynaptic inputs from the LVST to contralateral lumbar MNs.

\section{Spatial distribution of LVST-responsive} ipsilateral dCINs

Using the central canal and the midline as landmarks in the oblique plane, we determined the positions of all ipsilateral dCINs that were recorded and analyzed in the transverse plane of T7 $(n=83), \mathrm{L} 1$ $(n=87)$, L2 $(n=163)$, and L5 $(n=149)$, as described in Materials and Methods. Figure 6 shows for each segment the locations of the LVST-responsive dCINs (green circles) and LVST-unresponsive dCINs (red circles) and corresponding cumulative plots along the dorsoventral axis. In all four segments, most ipsilateral dCINs were located ventral to the midpoint of the central canal, with a spatial distribution similar to that reported previously for dCINs in the mouse embryo (Nissen et al., 2005) and neonate (Szokol et al., 2011). A striking feature in all four segments was the pronounced skewing of nonresponsive and responsive dCINs to dorsal and ventral locations, respectively. In T7 and L5, in which nearly all dCINs responded to activation of the LVST, the few nonresponsive dCINs were all restricted to approximately the dorsalmost third of the range (Fig. $6 A, D)$. In $\mathrm{L} 1$ and $\mathrm{L} 2$, in which the responsive dCINs were the minority, the unresponsive dCINs dominated the dorsal half of the range (Fig. $6 B, C$ ). The LVST-responsive and -nonresponsive dCIN populations had clearly distinct cumulative distributions along the dorsoventral axis.

To determine whether the segmentally specialized spatial pattern of LVST inputs onto ipsilateral dCINs was specific to this dCIN population, we also assessed the pattern of LVST inputs onto contralateral dCINs (T7, $n=145$; L1, $n=87$; L2, $n=148$; $\mathrm{L} 5, n=142$ ) in a total of 20 animals ( $n=6$ animals for T7, L2, and $\mathrm{L} 5, n=4$ for L1). Responsive contralateral dCINs were found in all four segments, and the segment-specific proportions followed the same pattern as for ipsilateral dCINs, with high proportions in T7 and L5 and low proportions in L1 and L2 (Table 1). The spatial distribution of nonresponsive and responsive contralateral dCINs was remarkably similar to that of ipsilateral dCINs (Fig. 6A-D), with clear skewing of nonresponsive dCINs toward dorsal positions in all four segments (Fig. $6 E-H$ ) and a pronounced ventral restriction of responsive contralateral dCINs in L1 and L2 (Fig. 6F,G). How this pattern is established in the 


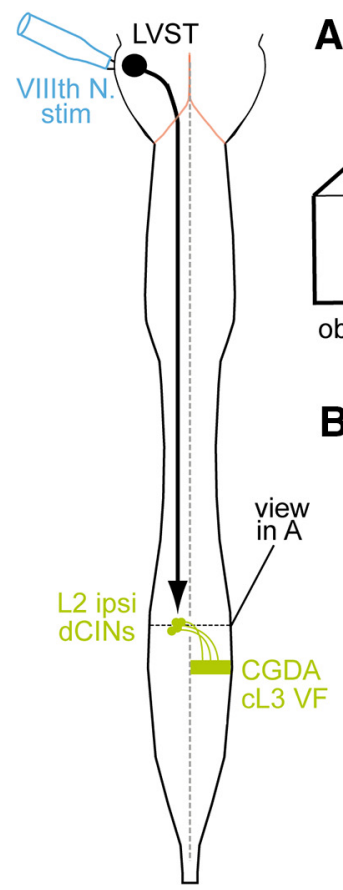

C

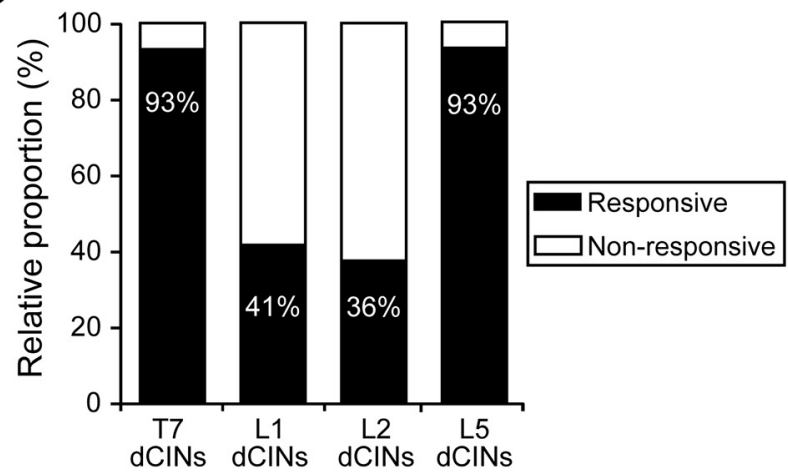

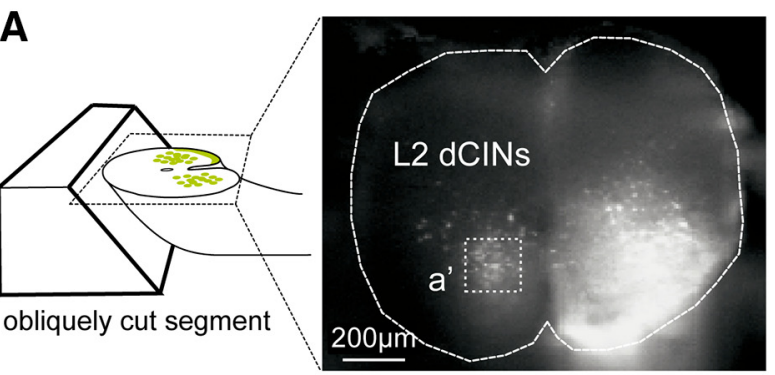

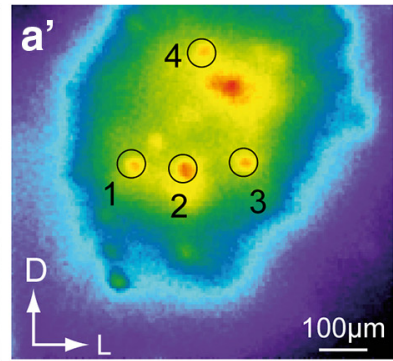

B

$$
\text { T7 ipsi dCINs }
$$

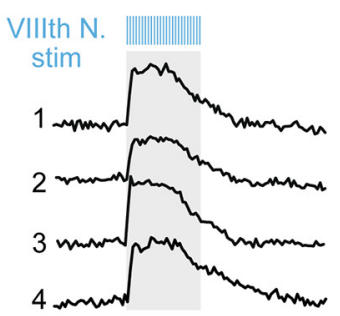

$$
\text { L2 ipsi dCINs }
$$

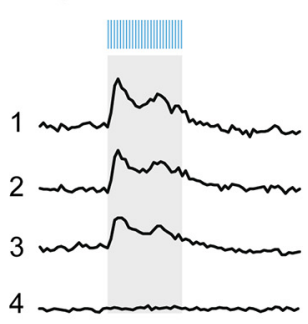

\section{L5 ipsi dCINs}

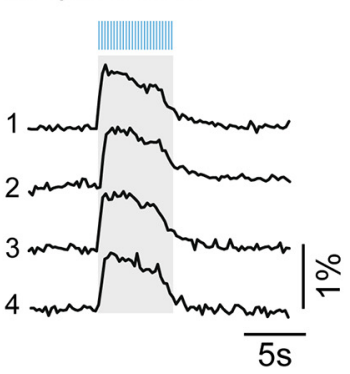

D

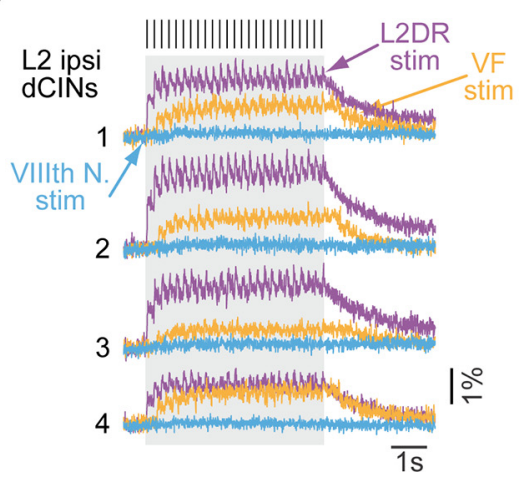

Figure 4. Optical recording of LVST-mediated synaptic inputs to ipsilateral dCINs. At the left is a schematic drawing of a preparation showing VIIIth nerve stimulation and retrograde labeling of ipsilateral L2 dCINs with CGDA applied at the L2/L3 border of the VF. A, The left shows a schematic drawing of the obliquely cut spinal cord showing the location of CGDA-labeled dIINs and dCINs as seen from the cut surface. The middle shows a black-and-white fluorescence photomicrograph of the obliquely cut face showing labeled dIINs (descending ipsilateral interneurons, right side) and dCINs (left side). Dorsal is up. The right shows at higher magnification the boxed area labeled in the middle (labeled a'), as a pseudocolor fluorescence image of CGDA-labeled dCINs, with fluorescence intensity coded with a rainbow color lookup table. ROls are placed over four dCINs as an example of where calcium waveforms are recorded. D, Dorsal; L, lateral. $\boldsymbol{B}$, Calcium response waveforms recorded at 4 fps from four individual T7 (left), L2 (middle), and L5 (right) dCINs in response to electrical stimulation of the ipsilateral VIIIth nerve (VIIIth N. stim). Gray bars represent the $5 \mathrm{~s}$, 5 Hz stimulation train. C, Comparison of average proportions of LVST-responsive and LVST-nonresponsive ipsilateral dCINs in T7, L1, L2, and L5. D, LVST-nonresponsive dCINs (in L2 shown here) respond to other inputs, including from the same segment DR afferents (L2 DR stim, purple responses) and general descending inputs coursing in the VF (VF stim, orange responses). Calcium responses to $5 \mathrm{~s}, 5 \mathrm{~Hz}$ stimulation train (gray bars). ipsi, Ipsilateral.

Table 1. Numbers of LVST-responsive and LVST-nonresponsive dCINs recorded on the two sides of the spinal cord (relative to the stimulated VIIIth nerve) in different

\begin{tabular}{|c|c|c|c|c|c|c|c|c|}
\hline \multirow[b]{2}{*}{ Segment } & \multicolumn{4}{|c|}{ Ipsilateral } & \multicolumn{4}{|c|}{ Contralateral } \\
\hline & $\mathrm{T} 7$ & L1 & L2 & $\mathrm{L} 5$ & $\mathrm{~T} 7$ & $\mathrm{~L} 1$ & L2 & $\mathrm{L} 5$ \\
\hline Animals $(n)$ & 4 & 4 & 6 & 6 & 6 & 4 & 6 & 6 \\
\hline dCINs recorded $(n)$ & 83 & 87 & 163 & 149 & 145 & 87 & 148 & 142 \\
\hline dCINs responsive $(n)$ & 77 & 36 & 58 & 138 & 115 & 32 & 50 & 132 \\
\hline dCINs responsive (\%) & $92.8 \%$ & $41.4 \%$ & $35.6 \%$ & $92.6 \%$ & $79.3 \%$ & $36.8 \%$ & $33.8 \%$ & $93.0 \%$ \\
\hline
\end{tabular}
segments

contralateral dCIN population is unclear. It could involve either midline-crossing collaterals of the LVST axons or transmission via CINs (Fig. 6I). The latter alternative seems most likely, given the effects of mephenesin application on responses in contralateral MNs described above, but in either case, innervation needs to be selective for those contralateral dCINs that are located ventrally.

Thus, LVST-mediated inputs to ipsilateral and contralateral dCINs exhibit a segmental pattern in the thoracolumbar spinal cord, being numerically and spatially more restricted in L1 and L2 relative to T7 and L5.

Ventrally located L1 and L2 dCINs project to contralateral L5 The results presented above implicate a ventrally restricted subpopulation of dCINs in L1 and L2 as potential candidates for the mediation of crossed extension. To provide additional support that they actually project to the segments that contain the major- 
A

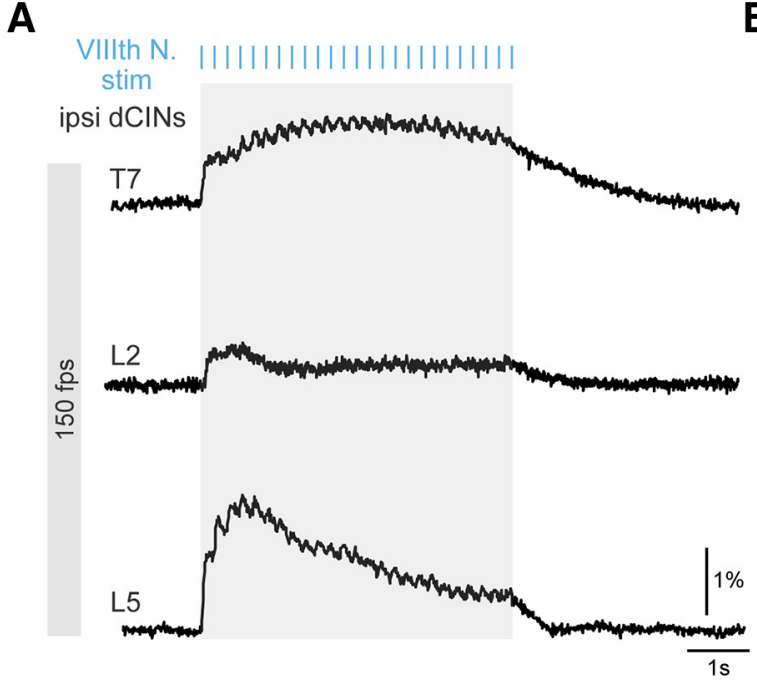

C

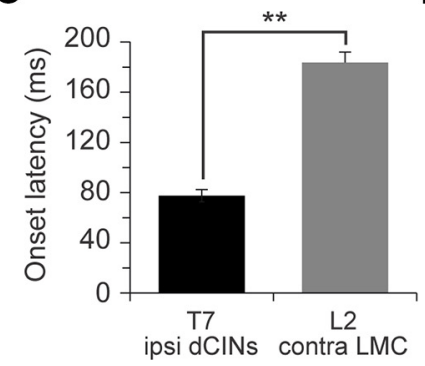

D

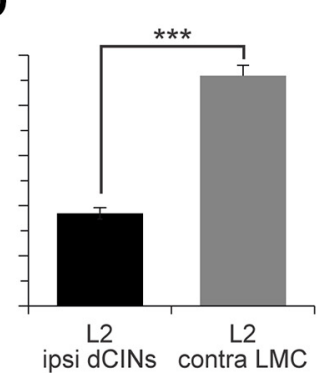

B

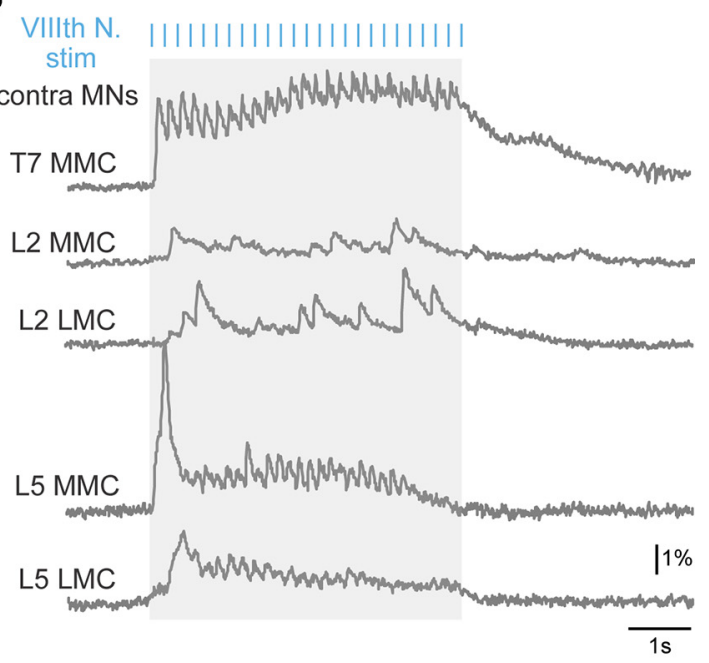

E

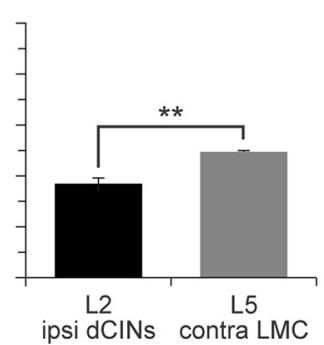

$\mathbf{F}$

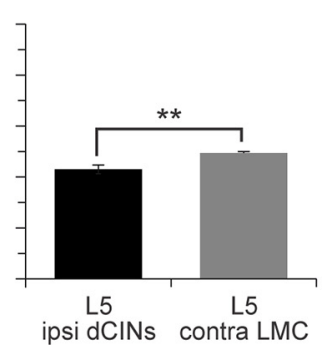

Figure 5. Onset latencies of LVST-mediated calcium responses in ipsilateral dCINs and contralateral MNs. $\boldsymbol{A}$, Responses recorded at $150 \mathrm{fps}$ in individual dCINs in T7, L2, and L5. B, Responses recorded in individual MNs at $150 \mathrm{fps}$ in the LMC and/or MMC of T7, L2, and L5. Gray bars represent the $5 \mathrm{~s}, 5 \mathrm{~Hz}$ stimulation train, and blue ticks indicate the individual stimuli. $C-F$, Comparison of average response onset latencies between various combinations of ipsilateral dCINs and contralateral LMC MNs in segments T7, L2, and L5. Bars and error bars represent averages (grand means of the averages for each preparation) and SEM (of the grand means). ${ }^{* *} p=0.01$, ${ }^{* *} p<0.001$, Mann-Whitney $U$ test $(\boldsymbol{C}, U=0, n=4, m=4 ; \boldsymbol{D}, U=0, n=6, m=4 ; \boldsymbol{E}, \boldsymbol{F}, \boldsymbol{U}=0, n=6, m=$ 3). contra, Contralateral; ipsi, ipsilateral.

ity of extensor MNs (L4 and L5 in the mouse; McHanwell and Biscoe, 1981; Cowley and Schmidt, 1994; Bácskai et al., 2014), we performed sequential retrograde labeling using two different conjugated dextran-amines to distinguish dCINs that project different distances ( $n=4$; Fig. 7). One dextran-amine was applied unilaterally to the border between L4 and L5, and then, after several hours of incubation, the other was applied unilaterally to the border between L2 and L3. Figure 7A shows the entire population of dCINs in L2, identified by the retrograde labeling from the L2/L3 border. Among these dCINs, we could distinguish those that projected at least as far as L5 by virtue of their double labeling from the L4/L5 border (Fig. $7 B$ ). In the same preparations, dCINs in L1 were labeled retrogradely from the L4/L5 border but could not be compared with the total population of L1 dCINs, because we did not also retrogradely label from the L1/L2 border; Fig. 7C). These were fewer in number than the L2 dCINs labeled retrogradely from the L4/L5 border, as expected from the normal drop off in dCIN number that occurs with distance. The labeling patterns demonstrated that a substantial proportion $(\sim 50 \%)$ of L2 dCINs located in the ventral region in which LVST-mediated inputs to dCINs are focused, as well as ventrally located dCINs in L1, project at least as far as L5 (Fig. 7D). Thus, in both L1 and L2, dCINs are positioned and project appropriately to mediate the pathway from LVST to contralateral extensor MNs. Because the other $50 \%$ of ventral L2 dCINs that did not project as far as L5 might project anywhere along the length of L3 and L4, it is not possible to conclude whether or not they might also be involved in mediating LVST inputs to hindlimb extensor MNs.

\section{Discussion}

We have shown that activating the LVST in the newborn mouse elicits a combination of monosynaptic and polysynaptic responses in thoracolumbar MNs, with responses in contralateral MNs mediated primarily by a polysynaptic pathway. We have further identified a segmentally patterned population of LVST-responsive ipsilateral dCINs interposed between the LVST and contralateral MNs. The hitherto unappreciated segment-specific allocation of LVST inputs to a dorsoventrally restricted dCIN subpopulation provides a selective channeling of vestibulospinal signals and a framework for understanding the role of dCINs in crossed vestibulospinal actions during balance, postural adjustments, and locomotion.

Our specific findings are as follows: (1) the LVST activates contralateral lumbar MNs at longer latencies than ipsilateral lumbar MNs; (2) mephenesin, which reduces primarily polysynaptic transmission, strongly reduces synaptic responses in contralateral MNs; (3) RV-mediated retrograde transsynaptic tracing from the hindlimb ankle extensor TS muscle group strongly labels only ipsilateral LVST neurons within a monosynaptic incubation period and contralateral LVST neurons predominantly within a later incubation period; (4) LVST-mediated responses in ipsilateral dCINs have shorter minimum and average latencies than LVST-mediated responses in contralateral MNs; (5) LVST-responsive ipsilateral dCINs show a differential rostrocaudal distribution in which the vast majority respond in thoracic (T7) and lower lumbar (L5) segments compared with only 40 and $35 \%$ in the upper lumbar L1 and L2 segments; (6) the 
A

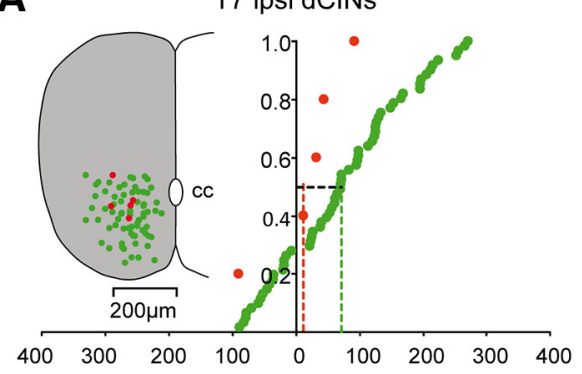

B

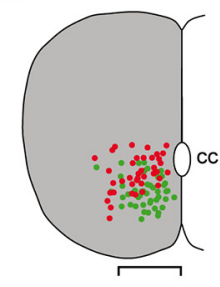

L1 ipsi dCINs

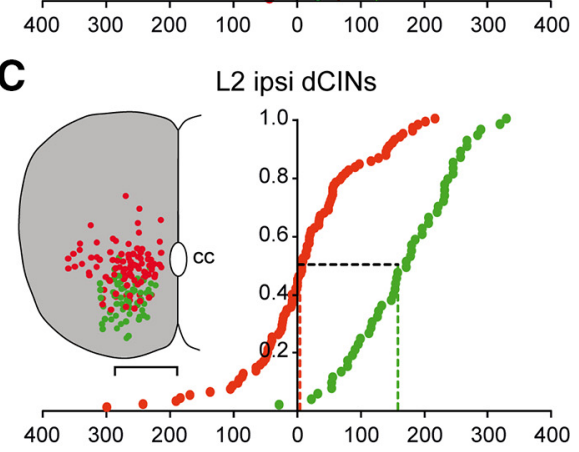

D

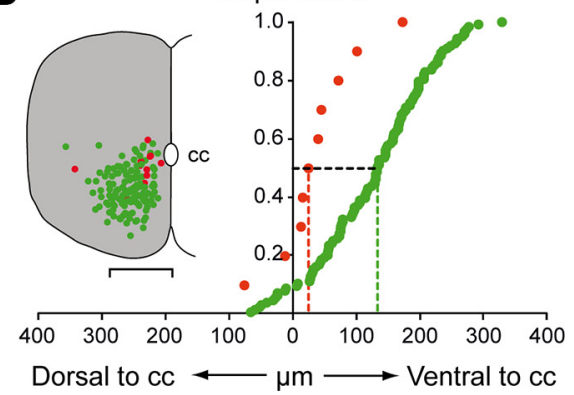

E

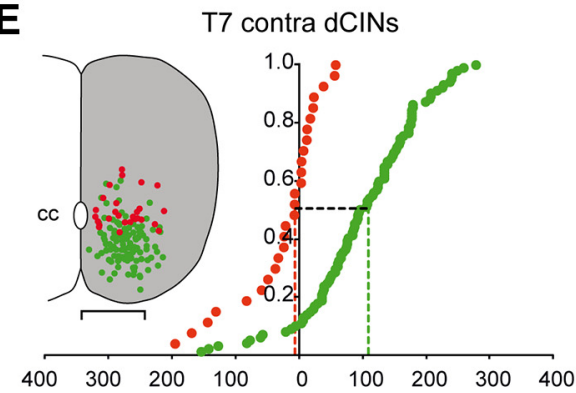

$\mathbf{F}$

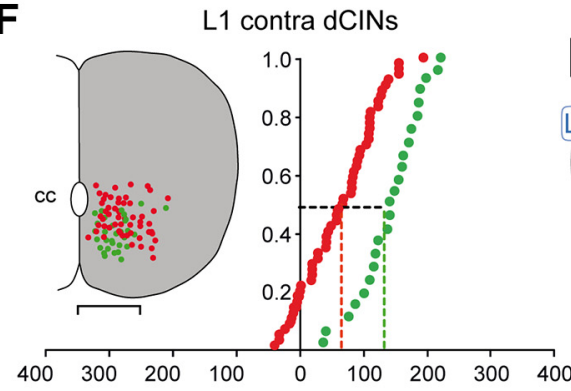

G

L2 contra dCINs
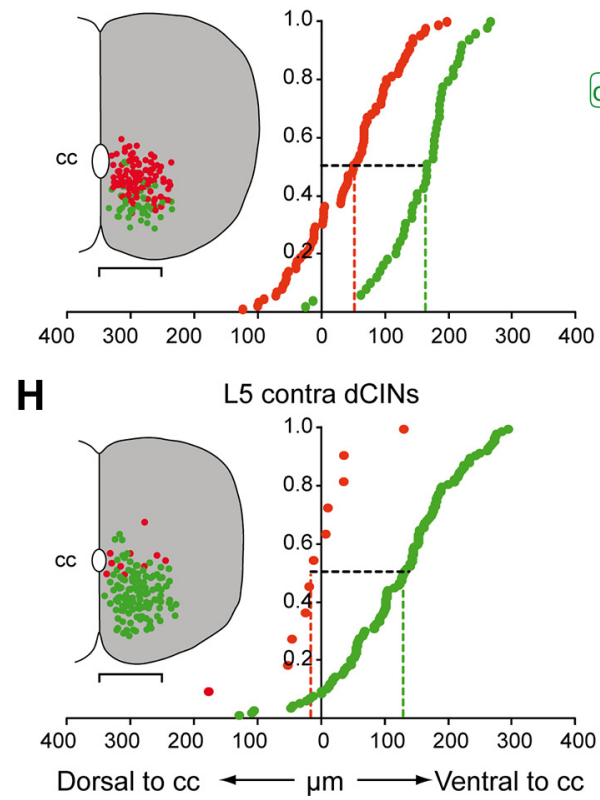

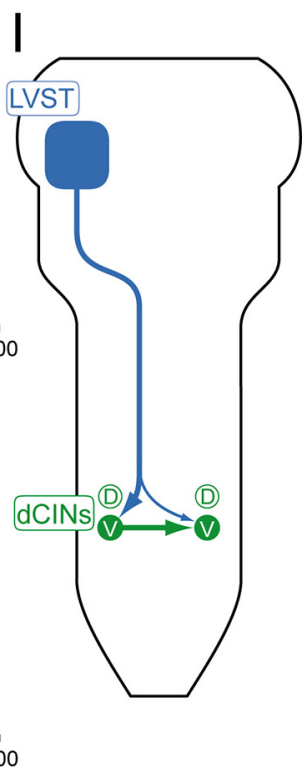

Figure 6. Comparison of the distributions of LVST-responsive and LVST-nonresponsive ipsilateral and contralateral dCINs in the transverse plane. $\boldsymbol{A}-\boldsymbol{H}$, Distributions of ipsilateral $(\boldsymbol{A}-\boldsymbol{D})$ and contralateral $(\boldsymbol{E}-\boldsymbol{H}) \mathrm{dCINs}$ in T7, L1, L2, and L5 are shown on the appropriate side of the transverse sections. Green and red dots represent LVST-responsive and -nonresponsive dCINs, respectively. Scale bar, $200 \mu \mathrm{m}$. Next to each section is a cumulative plot of distance along the dorsoventral axis [origin set at central canal (cc)], comparing the LVST-responsive (green) and LVST-nonresponsive (red) dCIN populations. The green and red dotted lines indicate the dorsoventral positions that mark the midpoints of each distribution. $\boldsymbol{I}, \mathbf{A}$ schematic diagram placed next to $\boldsymbol{B}, \boldsymbol{C}, \boldsymbol{F}$, and $\mathbf{G}$ to emphasize its relationship to the upper lumbar segments, illustrating two potential mechanisms whereby contralateral LVST-responsive dCINs in the upper lumbar segments may show the same ventral segregation as their ipsilateral counterparts. One involves a selective innervation of ventral contralateral dCINs (green circle, denoted by V on right side) by LVST axon collaterals that cross the midline (thin blue arrow). The other involves a selective innervation of ventral contralateral dCINs by ventral ipsilateral dCINs (green circle, denoted by V on left side), thus setting up a crossed, disynaptic activation via the LVST. A third mechanism (not illustrated here) is that a separate population of CINs (not necessarily dCINs), interposed between the LVST axons and the contralateral dCINs, selectively innervates the ventral contralateral dCINs. Selective transmission via ipsilateral ventral dCINs or another ipsilateral CIN population is most likely given the paucity of midline-crossing LVST collaterals observed in the adult mouse (Liang et al., 2014) and the effects of mephenesin application on responses in contralateral MNs (Fig. 2) but cannot be ruled out. contra, contralateral; ipsi, ipsilateral; D, dorsal dCINs (open green circles).

smaller population of LVST-responsive ipsilateral dCINs in upper lumbar segments have predominantly ventral locations and are segregated from more dorsal unresponsive dCINs, and many project as far as L5, in which hindlimb extensor MNs are concentrated; and (7) a similar segmentally modulated spatial distribution is exhibited by LVST-responsive contralateral dCINs. Some of these results have been reported previously in preliminary form (Perreault et al., 2009; Kasumacic et al., 2011).

\section{Polysynaptic pathway from the LVST to contralateral} thoracolumbar MNs

In the adult cat, the LVST makes both monosynaptic and polysynaptic connections onto ipsilateral and contralateral lumbar MNs, with polysynaptic connections predominating (Wilson and Yoshida, 1969; Grillner et al., 1970, Krutki et al., 2003). Excitatory and inhibitory polysynaptic connections are primarily to extensor and flexor MNs, respectively (Wilson and Yoshida, 1969; Grillner et al., 


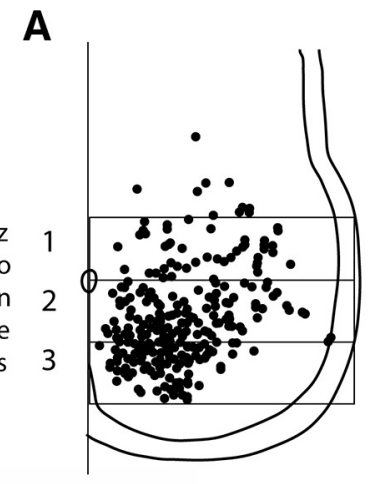

B

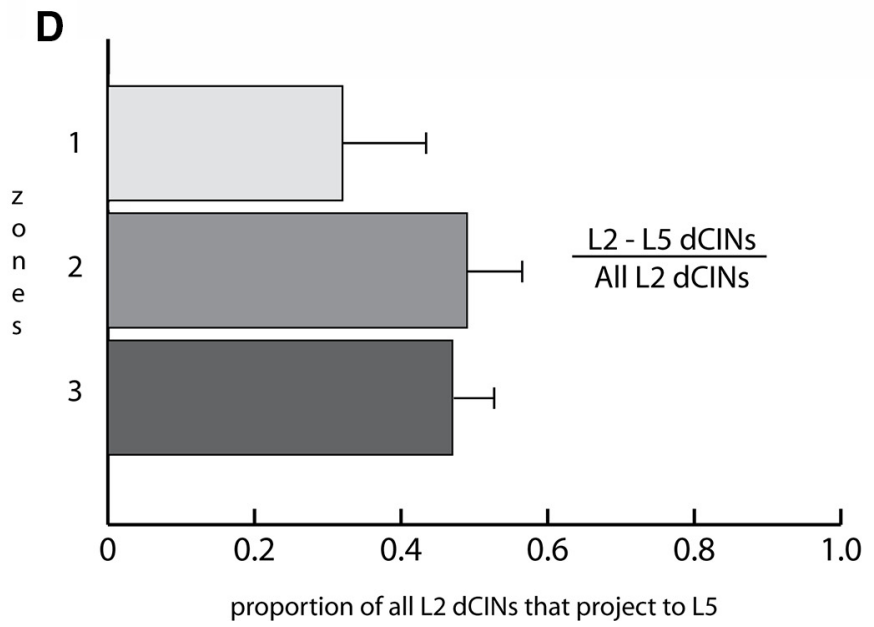

Figure 7. Comparative distributions of dCINs projecting different distances from upper lumbar segments. $A-C$, Plots of dCIN positions in compressed images of six transverse sections each, taken from the middle region of $L 2(\boldsymbol{A}, \boldsymbol{B})$ or $L 1(\boldsymbol{C})$, after sequential application of BDA to the L4/L5 border and RDA to the L2/L3 border. $A$, All L2 dCINs (retrogradely labeled from the L2/L3 border). $B$, The subset of $L 2 \mathrm{dCINs}$ that project axons to at least $L 5$ (retrogradely labeled from the $L 4 / L 5$ border). $C$, The dCINs in $L 1$ that project axons to at least $L 5$ (retrogradely labeled from the $L 4 / L 5$ border). The three equal dorsoventral zones ( $1-3$, with zones 2 and 3 dividing equally the region extending from the level of the central canal to the level of the most ventral d(IN) were used for assessing cell counts along the dorsoventral axis. Note that most dCINs projecting from $L 2$ to at least $L 5$ are in zones 2 and 3 , and most dCINs projecting from $L 1$ to at least $L 5$ are in zone 3. D, Proportions of $L 2 \mathrm{dCINs}$ projecting to $L 5$ in each of the three zones. Note that close to $50 \%$ of L2 dCINs in each of the two zones in the ventral horn (zones 2 and 3) project at least as far as L5. Error bars indicate SDs.

1970, Krutki et al., 2003), and LVST axon terminals are found in the ipsilateral lamina VIII and medial parts of ipsilateral lamina VII (Nyberg-Hansen and Mascitti, 1964; Erulkar et al., 1966), with few terminals seen contralaterally (Erulkar et al., 1966; Kuze et al., 1999). A similar picture has been reported recently in the adult mouse, in which only a few LVST terminals encroach on ipsilateral MNs, and only a very few are found contralaterally in the lumbar cord (Liang et al., 2014). Thus, dCINs, which densely populate lamina VIII and medial lamina VII (Bannatyne et al., 2003, Nissen et al., 2005), are prime candidates for mediating the polysynaptic connections onto contralateral MNs.

Our results show that the LVST has a widespread, polysynaptic influence on contralateral trunk and hindlimb MNs. We have presented several lines of evidence that polysynaptic inputs from the LVST specifically to contralateral extensor MNs in lower lumbar segments are mediated to a large extent by a ventrally restricted subpopulation of ipsilateral dCINs in the upper lumbar segments. Approximately half of these ventrally restricted LVSTresponsive dCINs project to L5 or beyond, and our previously published study using retrograde tracing with RV (Coulon et al., 2011) shows that dCINs in the same ventral region of upper lumbar segments are presynaptic to TS MNs. The channeling of LVST-mediated inputs through a spatially restricted dCIN subpopulation suggests a segment-specific modular organization of dCINs potentially related to functional motor output, in this case, the coordination of hindlimb extensor and trunk musculature during crossed vestibulospinal actions.

Two points are important to emphasize. First, reversible functional manipulation of the LVST-responsive dCINs in upper lumbar segments will be required to definitively prove that they mediate the crossed LVST input to hindlimb extensor MNs. This is technically challenging, but options are beginning to emerge. Targeting the expression in these dCINs of optogenetic hyperpolarizing ion channels, either via monosynaptically restricted $\mathrm{RV}$ injected into extensor muscles or via transgenics (assuming that this dCIN subpopulation can be targeted genetically), would be the cleanest approach. Neither of these options is without problems. Retrograde tracing with $\mathrm{RV}$ would target all last-order INs presynaptic to the MNs in question, which would lack the necessary specificity. Transgenic targeting would require that the ventrally restricted dCIN subpopulation (or its progenitor pool) expresses one protein (or two, if intersectional transgenics were used) uniquely, so that the promoter of the respective gene(s) could be used to target the dCINs. However, it is unclear whether this dCIN subpopulation is uniquely identifiable by gene expression. Preliminary experiments (N.K. and J.C.G., unpublished observations) indicate that it includes but is not restricted to one of the known transcription factor-defined IN classes in the ventral horn, so it is probably a heterogeneous population.

Second, we have studied the neonate, whose patterns of synaptic connectivity may differ from those in the adult. However, there are several indications that some basic features of spinal cord circuit connections, including in descending projections, are established already by birth in mammals (Glover, 2000; Kasumacic et al., 2010; Perreault and Glover, 2013). Because the vestibular system is evolutionarily ancient, it is not unlikely that core elements, such as the wiring of the crossed connections that reinstate balance after perturbations of head and body position, have been strongly selected for and are therefore established through highly determinate developmental mechanisms operating during fetal life.

\section{Segmental differences in the proportion and location of LVST-responsive dCINs}

The numerical and spatial restriction of responsive dCINs in L1 and L2 relative to T7 and L5 suggests a functional relationship between the segmental addresses of dCINs and the channeling of 


\section{Crossed vestibulospinal activation of thoracolumbar motoneurons via dCINs}
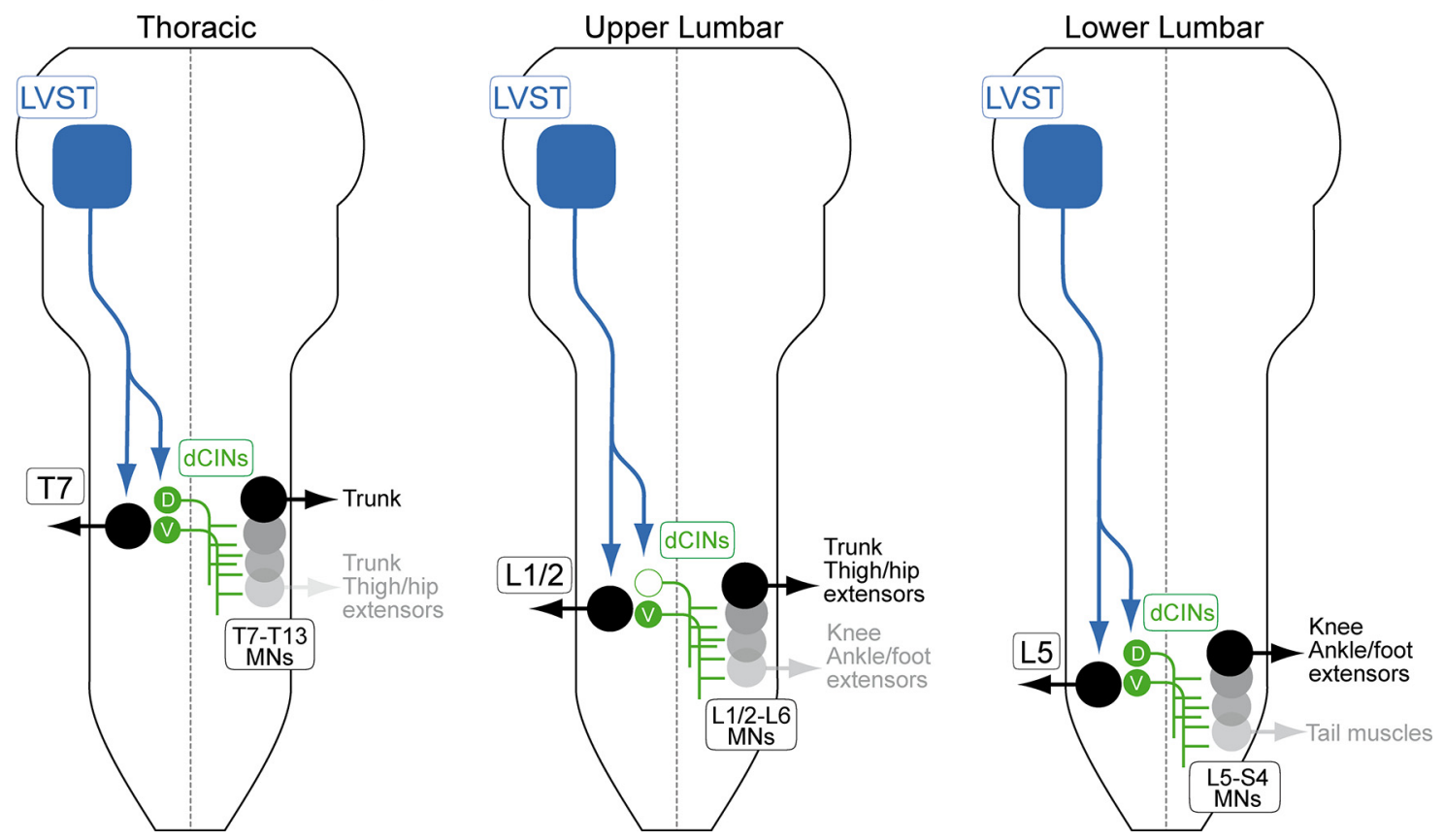

Figure 8. Schematic diagram summarizing the principal synaptic connection pattern from LVST to spinal neurons demonstrated in this study. LVST axons project to ipsilateral MNs (except for MMC MNs in lumbar segments; Kasumacic et al., 2010) and to ipsilateral dCINs but in a segment-specific pattern in which the more ventral dCIN subpopulation (V) but not the dorsal (D) is LVST responsive (filled vs open green dCINs, respectively) in the upper lumbar segments (L1/2). LVST-responsive dCINs in turn project to more caudal segments, which are shown with their contingent MN populations that innervate the indicated trunk, limb, and tail muscles.

vestibular information by the LVST to MNs and muscles. Of particular interest in this regard is that L2 and L5 are populated predominantly by flexor and extensor MNs, respectively (McHanwell and Biscoe, 1981; but see Cowley and Schmidt, 1994; Bácskai et al., 2014). It is known that extensor MNs represent the primary output of the LVST on the contralateral side (Grillner and Hongo, 1972, and see above) and that some dCINs in L2 are presynaptic to contralateral extensor MNs in L4 in the rat (Butt and Kiehn, 2003). We have raised the possibility that the restricted population of LVST-responsive dCINs in L1 and L2 is related specifically to hindlimb extensor MNs (Fig. 8). Seen in this light, perhaps the LVST-responsive dCIN populations in T7 and L5 are larger and more dispersed dorsoventrally because they are more heterogeneous with respect to their MN targets. For example, LVST-responsive $\mathrm{T} 7 \mathrm{dCINs}$ may not innervate hindlimb MNs at all but rather trunk MNs (because few are likely to project to lumbar segments anyway), and LVST-responsive L5 dCINs may innervate extensor MNs in more caudal lumbar segments and tail MNs in sacral segments, given the important role of the tail in balance (Fig. 8). Therefore, it will be important to identify the specific MN targets of the LVST-responsive dCINs in the various lumbar segments and place this into the context of the crossed, reciprocally organized extensor activation and flexor inhibition that characterizes vestibulospinal control of the hindlimbs, along with the associated activation of trunk musculature that is required to stabilize posture during limb movement. Moreover, because the LVST is capable of resetting the locomotor rhythm in cats (Russell and Zajac, 1979; Leblond et al., 2000), it will be equally important to investigate whether the LVSTresponsive dCINs include those that provide the crossed, reciprocal connections that characterize locomotion (Dougherty et al., 2013; Borgius et al., 2014), because this would represent a direct overlap between descending motor control and the functional organization of the locomotor central pattern generator.

\section{Developmental implications of differential segmental and dorsoventral distribution}

Examples abound of spatial segregation in the motor system, to the extent that it is now commonly believed that the specific anteroposterior and dorsoventral positions of MNs, premotor INs, and projection neurons are part of a developmental scheme by which the coarse strokes of motor connectivity are established (for review, see Glover, 2000). This has also been described previously for vestibular projection neurons (Glover, 2003). It is appealing in this regard that the specific LVST-responsive dCIN population in L1 and L2 is strikingly segregated from the nonresponsive $\mathrm{dCIN}$ population along the dorsoventral axis. Providing different spatial addresses to dCINs with different outputs would allow vestibulospinal and other axons presynaptic to the dCINs the opportunity to establish functionally appropriate polysynaptic pathways simply by targeting their collateral branches to different regions of the ventromedial spinal cord. Our results also suggest that this targeting may be generalized to include lumbar CIN-to-CIN connections, because the same ventral segregation is seen for LVST-responsive dCINs on the side contralateral to the LVST, and these are most likely activated via interposed CINs. Because these patterns of segregated activation have been set up by birth, it will be extremely interesting to follow the ingrowth of LVST axons during fetal life to see whether such axon targeting occurs and contributes to the segment-specific innervation of the ventral dCIN subpopulation.

\section{References}

Abzug C, Maeda M, Peterson BW, Wilson VJ (1974) Cervical branching of lumbar vestibulospinal axons. J Physiol 243:499-522. CrossRef Medline

Bácskai T, Rusznák Z, Paxinos G, Watson C (2014) Musculotopic organization of the motor neurons supplying the mouse hindlimb muscles: a quantitative study using Fluoro-Gold retrograde tracing. Brain Struct Funct 219:303-321. CrossRef Medline

Bannatyne BA, Edgley SA, Hammar I, Jankowska E, Maxwell DJ (2003) Net- 
works of inhibitory and excitatory commissural interneurons mediating crossed reticulospinal actions. Eur J Neurosci 18:2273-2284. CrossRef Medline

Borgius L, Nishimaru H, Caldeira V, Kunugise Y, Löw P, Reig R, Itohara S, Iwasato T, Kiehn O (2014) Spinal glutamatergic neurons defined by EphA4 signaling are essential components of normal locomotor circuits. J Neurosci 34:3841-3853. CrossRef Medline

Boulland JL, Lambert FM, Züchner M, Ström S, Glover JC (2013) A neonatal mouse spinal cord injury model for assessing post-injury adaptive plasticity and human stem cell integration. PLoS One 8:e71701. CrossRef Medline

Bras H, Gaytán SP, Portalier P, Zanella S, Pásaro R, Coulon P, Hilaire G (2008) Prenatal activation of 5-HT2A receptor induces expression of 5-HT1B receptor in phrenic motoneurons and alters the organization of their premotor network in newborn mice. Eur J Neurosci 28:1097-1107. CrossRef Medline

Brocard F, Vinay L, Clarac F (1999) Gradual development of the ventral funiculus input to lumbar motoneurons in the neonatal rat. Neuroscience 90:1543-1554. CrossRef Medline

Butt SJ, Kiehn O (2003) Functional identification of interneurons responsible for left-right coordination of hindlimbs in mammals. Neuron 38:953963. CrossRef Medline

Coulon P, Bras H, Vinay L (2011) Characterization of last-order premotor interneurons by transneuronal tracing with rabies virus in the neonatal mouse spinal cord. J Comp Neurol 519:3470-3487. CrossRef Medline

Cowley KC, Schmidt BJ (1994) Some limitations of ventral root recordings for monitoring locomotion in the in vitro neonatal rat spinal cord preparation. Neurosci Lett 171:142-146. CrossRef Medline

Dougherty KJ, Zagoraiou L, Satoh D, Rozani I, Doobar S, Arber S, Jessell TM, Kiehn O (2013) Locomotor rhythm generation linked to the output of spinal shox2 excitatory interneurons. Neuron 80:920-933. CrossRef Medline

Eide AL, Glover J, Kjaerulff O, Kiehn O (1999) Characterization of commissural interneurons in the lumbar region of the neonatal rat spinal cord. J Comp Neurol 403:332-345. CrossRef Medline

Erulkar SD, Sprague JM, Whitsel BL, Dogan S, Jannetta PJ (1966) Organization of the vestibular projection to the spinal cord of the cat. J Neurophysiol 29:626-664. Medline

Floeter MK, Lev-Tov A (1993) Excitation of lumbar motoneurons by the medial longitudinal fasciculus in the in vitro brain stem spinal cord preparation of the neonatal rat. J Neurophysiol 70:2241-2250. Medline

Glover JC (1995) Retrograde and anterograde axonal tracing with fluorescent dextran-amines in the embryonic nervous system. Neurosci Protoc 30:1-13.

Glover JC (2000) Neuroepithelial "compartments" and the specification of vestibular projections. Prog Brain Res 124:3-21. CrossRef Medline

Glover JC (2003) The development of vestibulo-ocular circuitry in the chicken embryo. J Physiol Paris 97:17-25. CrossRef Medline

Glover JC, Petursdottir G, Jansen JK (1986) Fluorescent dextran-amines used as axonal tracers in the nervous system of the chicken embryo. J Neurosci Methods 18:243-254. CrossRef Medline

Grillner S, Hongo T (1972) Vestibulospinal effects on motoneurones and interneurones in the lumbosacral cord. Prog Brain Res 37:243-262. CrossRef Medline

Grillner S, Hongo T, Lund S (1970) The vestibulospinal tract. Effects on alpha-motoneurones in the lumbosacral spinal cord in the cat. Exp Brain Res 10:94-120. CrossRef Medline

Hongo T, Kudo N, Tanaka R (1971) Effects from the vestibulospinal tract on the contralateral hindlimb motoneurones in the cat. Brain Res 31:220 223. CrossRef Medline

Huang Y, Goshgarian HG (2009) Identification of the neural pathway underlying spontaneous crossed phrenic activity in neonatal rats. Neuroscience 163:1109-1118. CrossRef Medline

Juvin L, Morin D (2005) Descending respiratory polysynaptic inputs to cervical and thoracic motoneurons diminish during early postnatal maturation in rat spinal cord. Eur J Neurosci 21:808-813. CrossRef Medline

Kasumacic N, Glover JC, Perreault MC (2010) Segmental patterns of vestibular-mediated synaptic inputs to axial and limb motoneurons in the neonatal mouse assessed by optical recording. J Physiol 588:4905-4925. CrossRef Medline

Kasumacic N, Glover JC, Perreault MC (2011) Vestibulospinal inputs to thoracolumbar commissural interneurons in the neonatal mouse assessed with optical recording. Soc Neurosci Abstr 41:809.03.

Kasumacic N, Glover JC, Perreault MC (2012) Vestibular-mediated synaptic inputs and pathways to sympathetic preganglionic neurons in the neonatal mouse. J Physiol 590:5809-5826. CrossRef Medline

Krutki P, Jankowska E, Edgley SA (2003) Are crossed actions of reticulospinal and vestibulospinal neurons on feline motoneurons mediated by the same or separate commissural neurons? J Neurosci 23:8041-8050. Medline

Kuze B, Matsuyama K, Matsui T, Miyata H, Mori S (1999) Segment-specific branching patterns of single vestibulospinal tract axons arising from the lateral vestibular nucleus in the cat: a PHA-L tracing study. J Comp Neurol 414:80-96. CrossRef Medline

Leblond H, Menard A, Gossard JP (2000) Bulbospinal control of spinal cord pathways generating locomotor extensor activities in the cat. J Physiol 525:225-240. CrossRef Medline

Lev-Tov A, Pinco M (1992) In vitro studies of prolonged synaptic depression in the neonatal rat spinal cord. J Physiol 447:149-169. CrossRef Medline

Liang H, Bácskai T, Watson C, Paxinos G (2014) Projections from the lateral vestibular nucleus to the spinal cord in the mouse. Brain Struct Funct 219:805-815. CrossRef Medline

Lund S, Pompeiano O (1968) Monosynaptic excitation of alpha motoneurones from supraspinal structures in the cat. Acta Physiol Scand 73:1-21. CrossRef Medline

McHanwell S, Biscoe TJ (1981) The localization of motoneurons supplying the hindlimb muscles of the mouse. Philos Trans R Soc Lond B Biol Sci 293:477-508. CrossRef Medline

Nissen UV, Mochida H, Glover JC (2005) Development of projectionspecific interneurons and projection neurons in the embryonic mouse and rat spinal cord. J Comp Neurol 483:30-47. CrossRef Medline

Nyberg-Hansen R, Mascitti TA (1964) Sites and mode of termination of fibers of the vestibulospinal tract in the cat. An experimental study with silver impregnation methods. J Comp Neurol 122:369-383. CrossRef Medline

Perreault MC, Glover JC (2013) Glutamatergic reticulospinal neurons in the mouse: developmental origins, axon projections, and functional connectivity. Ann N Y Acad Sci 1279:80-89. CrossRef Medline inelevel0

Perreault MC, Kasumacic N, Glover JC, Szokol K (2009) Bulbospinal control of lumbar motor networks in the neonatal mouse. 31st International Symposium of the Research Group of the Central Nervous System, Montreal, Canada, May.

Rose RD, Collins WF 3rd (1985) Crossing dendrites may be a substrate for synchronized activation of penile motoneurons. Brain Res 337:373-377. CrossRef Medline

Russell DF, Zajac FE (1979) Effects of stimulating Deiters' nucleus and medial longitudinal fasciculus on the timing of the fictive locomotor rhythm induced in cats by DOPA. Brain Res 177:588-592. CrossRef Medline

Stokke MF, Nissen UV, Glover JC, Kiehn O (2002) Projection patterns of commissural interneurons in the lumbar spinal cord of the neonatal rat. J Comp Neurol 446:349-359. CrossRef Medline

Szokol K, Perreault MC (2009) Imaging synaptically mediated responses produced by brainstem inputs onto identified spinal neurons in the neonatal mouse. J Neurosci Methods 180:1-8. CrossRef Medline

Szokol K, Glover JC, Perreault MC (2011) Organization of functional synaptic connections between medullary reticulospinal neurons and lumbar descending commissural interneurons in the neonatal mouse. J Neurosci 31:4731-4742. CrossRef Medline

Vinay L, Cazalets JR, Clarac F (1995) Evidence for the existence of a functional polysynaptic pathway from trigeminal afferents to lumbar motoneurons in the neonatal rat. Eur J Neurosci 7:143-151. CrossRef Medline

Wilson VJ, Yoshida M (1969) Comparison of effects of stimulation of Deiters' nucleus and medial longitudinal fasciculus on neck, forelimb, and hindlimb motoneurons. J Neurophysiol 32:743-758. Medline

Wilson VJ, Yoshida M, Schor RH (1970) Supraspinal monosynaptic excitation and inhibition of thoracic back montoneurons. Exp Brain Res 11: 282-295. CrossRef Medline

Ziskind-Conhaim L (1990) NMDA receptors mediate poly- and monosynaptic potentials in motoneurons of rat embryos. J Neurosci 10:125-135. Medline 